\title{
Bioquímica nos livros didáticos de Biologia: análise da presença de obstáculos epistemológicos
}

\author{
Biochemistry in high school biology textbooks: evaluation of \\ epistemological obstacles presence
}

\author{
Carolina Heyse Niebisch ${ }^{1}$ \\ Leila Cristina Aoyama Barbosa Souza ${ }^{2}$
}

\section{Resumo}

Tendo em vista a importância dos livros didáticos no contexto de sala de aula, o presente trabalho investigou a presença de obstáculos epistemológicos em conteúdos bioquímicos de quatro livros didáticos utilizados por professores da rede estadual de ensino de Florianópolis/SC. Para tanto foi avaliado, em uma análise qualitativa, o primeiro volume de cada obra, correspondente ao primeiro ano do ensino médio. A identificação e caracterização dos obstáculos de experiência primeira e conhecimento geral foi realizada nos conteúdos básicos de bioquímica (água, sais minerais, vitaminas, macromoléculas e metabolismo energético). Já a análise dos demais obstáculos (verbal, conhecimento unitário e pragmático, substancialista, realista e animista) foi realizada qualitativa e quantitativamente nos mesmos conteúdos, exceto o metabolismo energético. De acordo com os resultados obtidos foi possível notar a presença de todos os obstáculos epistemológicos nos materiais avaliado, com destaque para o obstáculo epistemológico substancialista. A presença destes obstáculos afeta negativamente a aquisição do conhecimento científico dos estudantes; portanto, seu diagnóstico pode vir a auxiliar os professores a lhes contornarem, reduzindo assim os entraves presentes no processo ensino-aprendizagem destes conteúdos nas escolas da região.

Palavras-chave: obstáculos epistemológicos; ensino de biologia; bioquímica; livros didáticos.

\section{Abstract}

Owing to the importance of textbooks in the classroom context, this study investigated the presence of epistemological obstacles in biochemical contents of four textbooks used by Florianópolis's teachers. For this purpose we evaluated the first volume of each collection from first year high school. The identification and characterization of the "first experience" and "general knowledge" obstacles was performed in all basic biochemical content (water, minerals, vitamins, macromolecules and energy metabolism). The analysis of the remaining obstacles (verbal, unitary and pragmatic knowledge, substantialism, realism and animism) was performed qualitatively and quantitatively in the same content, except the energy metabolism. According to the results, it was possible to detect all epistemological obstacles in materials evaluated, usually in the same contents. The obstacle most observed in all the textbooks was the substantialism. Whereas the presence of these barriers adversely affects the acquisition of scientific knowledge, this work can help teachers to overcome these obstacles reducing the barriers present in the teaching-learning of these contents in region schools.

Keywords: epistemological obstacles; science teaching; biochemistry; textbooks.

\footnotetext{
${ }^{1}$ Instituto Federal de Santa Catarina | carolinaheyse@gmail.com

2 Escola Técnica Estadual de Rondonópolis/MT | aoyama.leila@gmail.com
} 


\section{Introdução}

Os livros didáticos estão entre os principais recursos utilizados em sala de aula, tanto por professores quanto por alunos. Embora os Parâmetros Curriculares Nacionais (PCN) reforcem a necessidade da utilização de diferentes fontes de informação (BRASIL, 1997), em muitos casos isso não ocorre. Os relatos presentes na literatura indicam uma grande variação no que se refere à forma de utilização do livro didático. Em alguns casos, é utilizado como apoio instrumental para o desenvolvimento das atividades em sala de aula ou como apoio bibliográfico complementar (NETO e FRACALANZA, 2003; ROSA, 2013). Por outro lado, muitos docentes o utilizam como guia metodológico que vem a orientar desde os conteúdos a serem desenvolvidos, as atividades realizadas e até os processos de avaliação do ensino e não apenas como um apoio instrumental (FRISON et al., 2009). Assim, o livro didático "torna-se um dos principais responsáveis pela construção do conhecimento científico, exigindo maior rigor em sua linguagem, a fim de se evitar que o estudante crie uma visão distorcida da ciência." (AIRES et al., 2009: 1). Aliado a isto, sua grande utilização na prática docente evidencia a necessidade da utilização de material de alta qualidade.

Em assuntos científicos os livros didáticos podem ser redigidos com termos impróprios, figuras, analogias e metáforas equivocadas, em uma tentativa de facilitar o entendimento dos alunos o que pode vir a gerar entraves/bloqueios para a aquisição do conhecimento científico (MIRANDA e ARAÚJO, 2011). Estes bloqueios à aprendizagem são denominados obstáculos epistemológicos e podem ser classificados, de acordo com Bachelard (2005), em: a experiência primeira, o conhecimento geral, o obstáculo verbal, o conhecimento unitário e pragmático, o obstáculo substancialista, o obstáculo realista e o obstáculo animista.

Segundo Bachelard (2005) a experiência primeira vem a ser o obstáculo inicial para a cultura científica e

é a experiência situada antes e acima da crítica; [...]; que subordina a prática científica ao efeito das imagens; que dá grande atenção ao que é natural; que aborda fenômenos complexos como se fossem fáceis; que tem a marca de um empirismo evidente (ANDRADE et al., 2002: 5).

Ao iniciar qualquer estudo, os indivíduos já apresentam um conjunto de ideias próprias sobre o como e o porquê as coisas são como são. Na escola, durante a aquisição de novos conhecimentos, os estudantes elaboram construções pessoais com base no que observam ao seu redor, em suas interações cotidianas com as pessoas que o rodeiam e com os meios de comunicação e não na explicação cientifica. Desta maneira, são formados conceitos, ainda que errôneos do ponto de vista científico, que auxiliam o estudante em sua compreensão (ZAMORA, 2002).

A ausência da explicação científica, citada acima, pode vir a resultar em generalização. Esta generalização se enquadra no obstáculo do conhecimento geral e é decorrente da carência de entendimento claro sobre um tema, o que dificulta o interesse por seu aprofundamento e/ou questionamento. Andrade et al. (2002) classificam o conhecimento geral como vago, imobilizador de pensamento uma vez que fornece respostas muito fixas, seguras, vagas e gerais para qualquer questionamento. Na perspectiva da educação, estes obstáculos devem ser levados em conta uma vez que:

Os professores de ciências imaginam que o espírito [científico] começa como uma aula, que é sempre possível reconstruir uma cultura falha pela repetição da lição, que se pode fazer entender uma demonstração 
repetindo-a ponto por ponto. Não levam em conta que o adolescente entra na aula de física com conhecimentos empíricos já constituídos: não se trata, portanto, de adquirir uma cultura experimental, mas sim de mudar de cultura experimental, de derrubar os obstáculos já sedimentados pela vida cotidiana (BACHELARD, 2005: 23).

O obstáculo verbal se caracteriza por uma falsa explicação causada pelo uso de uma só palavra explicativa bem como a associação de uma palavra concreta a uma abstrata auxiliar do pensamento (BACHELARD, 2005). No geral, os exemplos apresentados por Bachelard (2005) para este tipo de obstáculo são relacionadas ao uso indevido de imagens, analogias e metáforas.

O obstáculo chamado conhecimento pragmático utiliza como princípio de explicação a procura de um caráter utilitário do fenômeno (BACHELARD, 2005). Já o obstáculo substancialista, "pode ser em parte oriundo do materialismo promovido pelo uso de imagens ou da atribuição de qualidades aos fenômenos" (GOMES e OLIVEIRA, 2007: 98). Por fim, o obstáculo animista é caracterizado pela atribuição de vida ou de características humanas para explicar substâncias ou fenômenos científicos (BACHELARD, 2005).

Em decorrência da importância do livro didático no contexto escolar, a seleção (e indicação) dos livros a serem utilizados nas escolas é realizada pelo Programa Nacional do Livro Didático (PNLD) e pelo Programa Nacional do Livro Didático para o Ensino Médio (PNLEM) levando em conta a avaliação de diferentes parâmetros. Contudo, a presença de obstáculos epistemológicos não se encontra entre os parâmetros eliminatórios no edital de convocação para o processo de inscrição e avaliação de obras didáticas incluindo o PNLD 2015 - Edital de convocação 01/2013-CGPLI (BRASIL, 2014a). Em decorrência disso, diversos autores ao avaliarem as obras didáticas do ensino médio descrevem a incidência de obstáculos epistemológicos em diferentes áreas de ciências (ANDRADE et al., 2002, PIRES, 2011, MIRANDA e ARAÚJO, 2011, TAUCEDA et al., 2011; STADLER et al., 2012).

A bioquímica foi selecionada como campo de estudo uma vez que seus conteúdos são bastante propícios ao aparecimento dos obstáculos epistemológicos por ser um campo amplo, interdisciplinar e passível de contextualização (PIRES, 2011). Outro ponto importante que pode acarretar no desenvolvimento de obstáculos epistemológicos consiste no fato da disciplina englobar diversos assuntos atuais (como clonagem, organismos geneticamente modificados, células-tronco, entre outros), muitas vezes exibidos em meios de comunicação de forma equivocada e/ou discutidos sem o conhecimento científico adequado. Por isso a bioquímica pode ser considerada "um nicho temático muito rico e promissor para abordagens interdisciplinares, contextualizadas social e experimentalmente" (FRANCISCO e FRANCISCO JUNIOR, 2010: 1). No entanto, os conteúdos bioquímicos presentes nos livros de ciências muitas vezes são apresentados de forma descontextualizada. Além disso, na tentativa de facilitar o entendimento, a transposição do conteúdo científico de bioquímica para uma forma didática pode resultar na ocorrência de obstáculos epistemológicos, como já registrado na literatura (FRANCISCO JUNIOR e JUNIOR, 2010).

Com base no que foi descrito acima é possível notar a importância da avaliação dos livros didáticos quanto à presença destes obstáculos. Sendo assim, o objetivo deste trabalho é descrever e caracterizar os obstáculos presentes, bem como a proposta de alternativas metodológicas que podem vir a auxiliar os professores a contornarem tais problemas com vistas à redução dos entraves presentes no processo ensino-aprendizagem. Este trabalho não pretende, de forma alguma, avaliar e comparar a qualidade dos livros didáticos; mas 
indicar aos professores que utilizam estes materiais onde os há a presença destes obstáculos e como tratá-los para evitar o desenvolvimento de obstáculos epistemológicos.

\section{Procedimentos metodológicos}

Para o desenvolvimento deste trabalho foi utilizada pesquisa aplicada do tipo qualitativa, baseada na análise documental de livros didáticos a fim de descrever e/ou caracterizar a presença de obstáculos epistemológicos (BACHELARD, 2005). A pesquisa de abordagem qualitativa valoriza "não a quantificação ou descrição dos dados recolhidos, mas a importância das informações que podem ser geradas a partir de um olhar cuidadoso e crítico das fontes documentais" (SILVA et al., 2009: 4556).

Primeiramente foi realizada a seleção dos livros didáticos, a qual seguiu os seguintes critérios:

- Livros de biologia do primeiro ano do ensino médio;

- Indicação (aprovação) no Guia de Livros Didáticos para o Ensino Médio - PNLD 2015/Biologia (BRASIL, 2014b);

- Utilização por professores atuantes em escolas públicas do município de Florianópolis/SC no ano de 2015;

- Disponibilidade para a pesquisa.

Com base nos critérios supracitados, dos 9 livros aprovados pelo PNLEM, foram selecionados quatro livros de biologia descritos na tabela 1. Para cada livro foi atribuído uma codificação a fim de facilitar a referência dos mesmos no decorrer do texto. Todos os livros analisados são seriados sendo compostos por três volumes. Em todos os casos, o primeiro volume foi selecionado para as análises, uma vez qu é neste volume que se encontram os conteúdos básicos de bioquímica (água, sais minerais, vitaminas, macromoléculas e metabolismo energético).

Tabela 1: Dados bibliográficos dos livros analisados.

\begin{tabular}{ccccc}
\hline Sigla & Título & Edição & Autor (es) & Editora \\
\hline A & Bio & $2^{\text {a }}$ & $\begin{array}{c}\text { Sônia Lopes } \\
\text { Sergio Rosso }\end{array}$ & Saraiva \\
\hline B & Ser protagonista & $2^{\text {a }}$ & $\begin{array}{c}\text { Márcia Regina Takeuchi } \\
\text { Tereza Costa Osorio }\end{array}$ & SM \\
\hline C & Biologia hoje & $2^{\text {a }}$ & $\begin{array}{c}\text { Sérgio de Vasconcelos } \\
\text { Linhares }\end{array}$ & Ática \\
\hline D & Biologia & $1^{\text {a }}$ & $\begin{array}{c}\text { César da Silva Júnior } \\
\text { Sezar Sasson } \\
\text { Nelson Caldini Júnior }\end{array}$ & Saraiva \\
\hline
\end{tabular}

A análise dos livros foi realizada baseada nos trabalhos de Stadler et al. (2012). Após a triagem dos capítulos referentes aos conteúdos bioquímicos, foi verificada a integração entre as linguagens cotidiana e científica, visto que a ausência de integração pode acarretar no aparecimento do obstáculo da experiência primeira e do conhecimento geral. Além disso, foi examinada a fragmentação e a contextualização dos conteúdos bioquímicos. 
Nesta etapa foram analisados, na íntegra, os capítulos relacionados aos temas: água, sais minerais, vitaminas, macromoléculas (lipídeos, carboidratos, proteínas e ácidos nucléicos) e metabolismo energético.

A segunda etapa consistiu em determinar a presença dos obstáculos: verbal, conhecimento unitário e pragmático, substancialista, realista, animista nos textos e nas imagens referentes aos três primeiros supracitados. Nesta etapa, os principais obstáculos foram quantificados, descritos, analisados, classificados e discutidos.

\section{Resultados e discussão}

Inicialmente foi verificada a estrutura geral dos capítulos que contemplam os conteúdos bioquímicos visando analisar a presença dos obstáculos de experiência primeira e do conhecimento geral. A análise destes obstáculos não é consenso entre diferentes autores. Miranda e Araújo (2012) indicam que o obstáculo da experiência primeira é caracterizado pela linguagem falada e encontrado no diálogo de sala de aula, portanto não se apresenta na linguagem escrita. Em decorrência disso, os autores não incluem estas categorias em suas análises da presença de obstáculos em livros didáticos. Por outro lado, Stadler et al (2012) realizaram esta análise pautada na observação de como os conteúdos eram expostos aos alunos quanto à contextualização e a linguagem utilizada. De acordo com os autores, a simplificação e subjetividade inerente à transposição didática dos conhecimentos científicos podem se transformar em obstáculos epistemológicos que afetam o conhecimento científico desviando suas teorias e conteúdos.

$\mathrm{Na}$ aquisição de novos conhecimentos, os estudantes elaboram construções pessoais de um determinado assunto baseadas em diversos fatores como: conhecimentos prévios sobre o tema, o que observam ao seu redor, em suas interações cotidianas interpessoais e com os meios de comunicação. Com isso são formadas concepções que auxiliam o estudante na compreensão dos conceitos estudados, ainda que errôneos do ponto de vista científico (ZAMORA, 2002). Portanto, considerando que muitos professores e estudantes utilizam o livro didático como principal (se não única) fonte de consulta, neste trabalho foi realizada esta análise.

A forma de apresentação dos conteúdos bioquímicos nos livros didáticos foi bastante variada tanto no que se refere à distribuição quanto à localização dos conteúdos nos diferentes materiais didáticos. Na maioria das obras a linguagem cientifica foi apresentada de forma clara sem muitas simplificações. Porém algumas definições não estão corretas/completas ao longo dos textos; o que pode causar prejuízos para a obtenção do conhecimento científico. Como, por exemplo, a definição de lipídeos. O livro " $\mathrm{C}$ " indica como lipídeos os óleos e as gorduras. Já os livros "B" e "D" indicam diferentes tipos de lipídeos. Porém, os três livros citados não apresentam de forma clara quais moléculas compõem esta classe de macromoléculas, nem sua principal característica em comum. Esta constatação indica uma simplificação na explicação do termo, o que pode vir a gerar o obstáculo do conhecimento geral. E, ainda da experiência primeira uma vez que reforça o significado utilizado pelo senso-comum. O erro conceitual na definição dos lipídeos presentes nos livros textos já se encontra descrito na literatura. De acordo com Francisco e Junior (2010), dos seis livros didáticos de química aprovados pelo PNLEM de 2007, apenas dois deles apresentam uma definição satisfatória do termo. Neste ponto apenas o Livro "A" apresenta uma caracterização satisfatória deste grupo de macromoléculas. Segundo os 
autores "os lipídeos abrangem uma classe de compostos com estrutura muito variada que exercem diferentes funções biológicas. São substâncias fisicamente caracterizadas pela insolubilidade em água e solubilidade em solventes orgânicos" (Livro A, p. 188).

Ainda no que tange a linguagem, os termos científicos são apresentados geralmente destacados do texto (em negrito) em todas as obras avaliadas. Em todos os livros é dada ênfase na origem etimológica dos conceitos, sendo nos Livros " $C$ " e " $D$ " grande parte de forma parcial. Nunes (2013) indica o uso de uma investigação prévia relacionada à etimologia dos termos científicos para facilitar seu entendimento, ou seja, o estudo do significado dos prefixos e sufixos que compõe a terminologia. A aplicação deste tipo de estudo visa o melhor entendimento dos termos, de forma a superar os conhecimentos prévios relacionados a ele e reduzir a possibilidade do desenvolvimento de diferentes tipos de obstáculos epistemológicos. Contudo, a descrição incompleta da etimologia dos termos faz com que seja perdido o sentido desta descrição reforçando o desenvolvimento de possíveis obstáculos.

A maior parte da contextualização dos conteúdos científicos nas quatro obras avaliadas é dada ao longo do texto na forma de exemplificação (como no livro "B" em figuras). Cabe destacar aqui que a contextualização é rotineiramente entendida como exemplificação (FERNANDES e MARQUES, 2012), porém este é um tema polissêmico. Neste trabalho a contextualização é entendida como integração entre o conhecimento específico da disciplina e contextos mais amplos de significação como "o cotidiano do aluno, a(s) disciplina(s) escolar(es), a ciência (referência), o ensino e os contextos histórico, social e cultural" (KATO e KAWASAKI, 2011: 46). E, com base no observado nos livros é possível notar a importância da consulta a diferentes materiais que auxiliem a uma real integração entre os conteúdos científicos, escolares e cotidiano.

Embora os temas bioquímicos apresentem relação direta com o cotidiano (uma vez que trata da respiração, do que ocorre com os alimentos após a ingestão, ganho/perda de peso, as reações do organismo frente às atividades físicas, a fotossíntese, entre vários outros temas) nota-se que, na maioria dos casos, independentemente do livro avaliado, o texto não apresenta relações com o cotidiano dos alunos. Este fato está na contramão da realidade uma vez que os conteúdos bioquímicos dos livros didáticos apresentam ligação direta com o dia a dia de todas as pessoas. A aproximação com a realidade dos alunos é de suma importância para que os alunos tenham mais interesse nos assuntos estudados, bem como compreendam que os fenômenos estudados na biologia ocorrem também em suas atividades diárias e ao seu entorno. Os temas bioquímicos poderiam ser colocados de forma a enfatizar o que contém cada tipo de alimento e esta relação com o desenvolvimento de doenças ou com o ganho e/ou perda de peso, por exemplo. Desta forma, além de uma correta explicação dos fenômenos científicos é possível desfazer conceitos prévios sem bases científicas, superando o obstáculo da experiência primeira e da generalização.

Tendo em vista que estes obstáculos são caracterizados principalmente pela linguagem falada e encontrados no diálogo de sala de aula, para que eles sejam realmente superados há a necessidade dos professores tomarem conhecimento do que são e de como evitá-los.

Após a análise da estrutura geral, foi realizada a quantificação dos demais tipos de obstáculos presentes nos livros didáticos nos conteúdos de água, sais minerais, vitaminas e macromoléculas. Esta avaliação resultou nos dados apresentados na tabela 2. Sendo o 
obstáculo observado em maior número o substancialista e os observados em menor frequência o realista e o conhecimento unitário e pragmático.

Tabela 2: Quantificação dos diferentes tipos de obstáculos presentes em cada livro didático avaliado.

\begin{tabular}{cccccc}
\hline LivrolObstáculo & Verbal & $\begin{array}{c}\text { Conhecimento unitário e } \\
\text { pragmático }\end{array}$ & Substancialista & Realista & Animista \\
\hline A & 2 & 1 & 3 & 1 & 1 \\
B & 2 & 1 & 2 & 1 & 1 \\
C & 0 & 1 & 2 & 0 & 2 \\
D & 2 & 0 & 2 & 1 & 1 \\
\hline
\end{tabular}

Da mesma forma ao observado neste trabalho, a presença de obstáculos epistemológicos em livros didáticos já está bem relatada na literatura. Por exemplo, Miranda e Araújo (2012) observaram uma quantidade acentuada de obstáculos em quatro livros didáticos de química, sendo, ao todo, 31. O obstáculo realista foi observado em todas as obras avaliadas, o animista em três delas e os obstáculos substancialista e verbal em metade das obras.

Por outro lado, Stadler et al. (2012) ao avaliarem a presença de obstáculos epistemológicos em cinco livros didáticos de química, indicados pelo PNLEN 2012, observaram a presença de 23 obstáculos no total das obras. O obstáculo verbal foi o mais recorrente, aparecendo em todas as obras avaliadas (15 no total) em quantidades variáveis (1 a 6 obstáculos por obra). Além disso, os autores indicam que a não ocorrência do obstáculo animista nas obras avaliadas pode denotar uma preocupação dos autores em utilizar a linguagem científica na explicação dos fenômenos e a não atribuir características vivas a substâncias não vivas. Em contrapartida, nos livros utilizados esta análise a presença do obstáculo animista foi detectada em todos os materiais.

Também neste trabalho, a presença de alguns obstáculos específicos foi recorrente nas obras didáticas avaliadas. Como, por exemplo, quando tratado o assunto água, houve a definição simplista das ligações de hidrogênio, que pode vir a consolidar nos estudantes um entendimento errôneo sobre a ligação de hidrogênio. Tal fato pode se observar no livro "A", transcrito a seguir.

Por causa da atração entre cargas elétricas opostas, cada molécula de água tende a se unir umas as outras quatro de modo que um átomo de hidrogênio de uma molécula sempre se ligue ao átomo de oxigênio de outra molécula. Estas interações são denominadas ligações de hidrogênio (Livro A: 180).

O livro " $\mathrm{C}$ " demonstra este tipo de ligação apenas na forma de figura indicando que ela ocorre entre o oxigênio e hidrogênio (Livro C, p. 37), embora nenhuma menção a este tipo de ligação seja encontrada no corpo do texto. No livro "B" este conceito é apresentado da mesma forma (Livro B, p. 25), porém um pouco adiante no texto é mencionado que a ligação de hidrogênio não ocorre somente entre estes dois átomos (Livro B, p. 27). Já no livro D não é mencionada a estrutura tridimensional da molécula de água.

Este conceito é descrito nos livros "A", "B" e "C" de forma simplista. Uma alternativa a esta simplificação seria a descrição de que as ligações de hidrogênio ocorrem entre o 
hidrogênio e átomos mais eletronegativos como o flúor e nitrogênio, por exemplo. E não apenas entre hidrogênio e oxigênio. Além disso, este tipo de ligação não é estritamente observado em moléculas de água (NELSON e COX, 2008). Esta descrição simplificada pode acarretar no desenvolvimento do obstáculo do conhecimento geral, uma vez que é observada a generalização do termo.

Outro tipo de obstáculo que se enquadra neste exemplo é o substancialismo (BACHELARD, 2005), pois todos os conhecimentos sobre a ligação de hidrogênio são condensados em um mesmo objeto, neste caso em um único tipo de ligação. E, além disso, aparecem relacionados à tentativa de relacionar os conceitos científicos de forma simplificada com a intenção de facilitar o entendimento do conceito pelo aluno, impedindo que este realize abstração necessária à aquisição do conhecimento científico (STADLER et al., 2012). Para a aquisição do conceito científico deste tipo de ligação deve-se mencionar todos os possíveis átomos que participam deste tipo de ligação e o porquê ocorre esta interação. A descrição correta da ligação de hidrogênio é de suma importância no âmbito do entendimento da tensão superficial da água, de sua influência na estabilidade de diversas moléculas e de seu papel das reações metabólicas.

Quanto à explicação sobre as reações enzimáticas, um dos processos vitais na fisiologia dos seres vivos, três dos quatro livros avaliados indicam que a reação enzima-substrato ocorre de acordo com a teoria chave-fechadura. Os livros indicam que

A grande especificidade enzima-substrato está relacionada à forma tridimensional de ambos. Eles se encaixam perfeitamente como chaves em fechaduras. Essa teoria da atividade enzimática é denominada teoria da chave-fechadura (Livro A: 196, grifo nosso).

Assim, existe uma combinação entre o formato da enzima e o formato das substâncias sobre as quais ela age, como uma chave com sua fechadura (Livro B: 36).

O encaixe entre enzima e substrato somente ocorre se as formas das moléculas forem favoráveis, o que sugere um encaixe perfeito entre uma chave e uma fechadura (Livro D: 48, grifo nosso).

Entre os livros analisados apenas no livro "C" é apresentado outro modelo de interação enzima-substrato como descrito a seguir

Costuma-se dizer que a enzima está para o substrato assim como uma chave está para uma fechadura. Uma enzima, no entanto, é capaz de ajudar a reação de substratos semelhantes, contanto que eles participem do mesmo tipo de reação. A explicação para esta flexibilidade é dada pela capacidade da enzima modificar um pouco a forma do seu centro ativo, possibilitando o encaixe [...] Esta explicação é conhecida como modelo do encaixe induzido (Livro C: 36).

O mecanismo de chave-fechadura, complementariedade entre substrato e enzima, foi desenvolvido em 1894 e teve grande influência no desenvolvimento da bioquímica. Porém, com o passar do tempo e desenvolvimento da ciência observou-se que o processo não ocorre desta forma (NELSON e COX, 2008). Do ponto de vista bioquímico, esta ideia se encontra atualmente equivocada. O substrato e a enzima não apresentam encaixe perfeito. Caso houvesse este encaixe o complexo enzima-substrato seria termodinamicamente mais estável que os reagentes e os produtos da reação, impedindo a reação de ocorrer 
(FRANCISCO e JUNIOR, 2010; NELSON e COX, 2008). Por conseguinte, foi observado um erro conceitual em três dos quatro livros. Além disso, este pode acarretar no desenvolvimento do obstáculo do tipo realista, uma vez que é observada a "supervalorização das impressões táteis e visuais" (LOPES, 1992: 258 apud MIRANDA e ARAÚJO, 2012: 6) sobre fenômenos abstratos. Também este exemplo pode se caracterizar em obstáculo verbal a medida que é observada uma falsa explicação do encaixe enzimasubstrato causada pelo uso de palavras explicativas, neste caso uma falsa analogia.

O livro " $C$ ", embora traga uma definição mais científica da interação enzima-substrato, durante a explanação do tema é possível observar a presença de um obstáculo animista nesta descrição. Os autores indicam que uma enzima é capaz de "ajudar" as reações. Neste caso, o fenômeno é explicado pelos autores através de analogias com comportamentos humanos, o que caracteriza a presença deste tipo de obstáculo. A definição presente neste livro é válida cientificamente, porém sugere-se alterar a expressão "ajudar a reação" por termos científicos como "catalisar a reação".

Neste exemplo (assim como observado na explicação de energia de ativação) pode-se notar a importância do cuidado na utilização de analogias no ensino de ciências. De acordo com Bachelard (2005), o uso de analogias e metáforas está bastante ligado às concepções prévias dos estudantes e com isso, pode acarretar na formação e/ou desenvolvimento de obstáculos epistemológicos, como o verbal, o substancialista e o animista. O autor, contudo, não critica o uso destas analogias e metáforas desde que o conceito científico seja transmitido antes destes artifícios. Outros autores indicam a importância do uso de metáforas e analogias no ensino de ciências, justamente com o intuito de mobilizar os conhecimentos prévios dos estudantes, desde que este uso esteja acompanhado da indicação das limitações entre os conteúdos alvo e a analogia (BARBOSA et al., 2012; BERNARDINO et al., 2013).

Nos livros "A", "B" e "D" foi encontrado um exemplo de obstáculo animista cuja utilização é muito corriqueira. Ao descrever os tipos de colesterol os autores indicam que "a LDL é chamada de "mau colesterol". Já a HDL, chamada de "bom colesterol"" (Livro A: 191; Livro B: 32; Livro D: 39). Embora este assunto esteja presente nos livros "B" e "D", este aparece em forma de boxes separados do texto. No livro C o termo é só citado, porém o termo não e definido. Embora as lipoproteínas e a molécula de colesterol sejam a mesma nos dois casos e apenas exerçam funções diferentes. Pode-se notar claramente a atribuição de características humanas para explicar substâncias ou fenômenos científicos não vivos. Este exemplo caracteriza também o obstáculo tipo verbal, pois ocorre uma explicação errônea causada pelo uso de palavra explicativa.

Não foram observados erros conceituais na descrição das demais macromoléculas, porém quando abordado sobre os aminoácidos essenciais na dieta, o modo como foi descrito pode vir a causar prejuízos ao correto entendimento do assunto. Nos livros " $A$ ", " $B$ " e "C" uma classificação recorrente foi a denominação de aminoácidos essenciais aos aminoácidos não sintetizados pelo organismo. Embora esta classificação seja recorrente em diversos tipos de livros, ela pode induzir o estudante a pensar que os aminoácidos sintetizados pelo organismo não são essenciais. Porém, todos os aminoácidos são essenciais aos seres humanos. O termo "essenciais" é utilizado para se referir a necessidade de obtenção destes aminoácidos pela ingestão de alimentos. Portanto, esta descrição pode acarretar no desenvolvimento do obstáculo do conhecimento unitário e pragmático uma 
vez que atribui, de forma errônea, a qualidade de essencial apenas aos aminoácidos não biossintetizados.

\section{Considerações finais}

O presente trabalho demonstrou a presença de obstáculos epistemológicos em todos os materiais didáticos avaliados, fato possivelmente derivado da não utilização deste critério durante a análise, seleção e indicação dos livros didáticos pelo PNLEM. Todos os sete tipos de obstáculos epistemológicos foram encontrados.

A ocorrência destes obstáculos, por ser caracterizada como entrave a obtenção do conhecimento científico, pode vir a gerar um conhecimento parcial e/ou equivocado de conceitos, prejudicando o acompanhamento dos próximos conteúdos e gerando uma defasagem ao discente. Além disso, a presença destes obstáculos nos livros didáticos é preocupante, uma vez que muitos professores utilizam este material como guia metodológico em sala de aula. E, caso não seja detectada a presença dos obstáculos, estes serão repassados aos alunos reforçando a aquisição de um conhecimento equivocado. Neste ponto, é possível destacar a importância da formação de profissionais (tanto inicial quanto continuada) preparados para reconhecer e superar estes obstáculos com o rigor científico necessário a esta tarefa.

Convém destacar que o propósito deste trabalho consistiu em descrever e caracterizar os obstáculos presentes em quatro livros didáticos utilizados por professores em escolas de Florianópolis/SC e indicar, aos professores que utilizam destes materiais, onde são apresentados conteúdos equivocados. Para auxiliar o trabalho docente a proporcionar uma redução nos entraves presentes no processo ensino-aprendizagem destes conteúdos nas escolas da região. E não tem, de forma alguma, a intenção de avaliar a qualidade dos materiais.

Assim como evidenciou este trabalho, diversos relatos descritos na literatura indicam que a presença destes obstáculos é recorrente nos materiais destinados ao ensino médio brasileiro nos mais diversos conteúdos e disciplinas. Portanto fica claro a importância de trabalhos neste sentido para as demais obras recomendadas para utilização no ensino médio, a fim de reduzir ou até mesmo eliminar a incidência destes obstáculos ao desenvolvimento do conhecimento científico.

\section{Referências}

AIRES, A. JOANEZ; GUIMARÃES, ORLINEY M.; LARA DA SiLVA MOISÉS. Obstáculos epistemológicos no ensino de Química: Uma análise do livro didático público do Paraná. In: Congresso Paranaense de Educação de Química, 1, 2009, Londrina. Anais.

ANDRADE, B. L.; ZYLBERSZTAIN, A.; FERRARI, N. As analogias e metáforas no ensino de ciências à luz da epistemologia de Gaston Bachelard. Ensaio - Pesquisa em Educação em Ciências, v.2, n.2, p.1-11, 2002.

BACHELARD, G. A formação do espírito científico: contribuição para uma psicanálise do conhecimento. $5^{\circ}$ reimpressão, Rio de Janeiro: Contraponto, 2005, 309 p. 
BARBOSA, J. U.; LEAL, M. C.; ROSSI, S. Q.; DIA, T. N.; FERREIRA, K. A.; OliveIRA, C. P. Analogias para o ensino de bioquímica no nível médio. Ensaio - Pesquisa em Educação em Ciências, v. 14, n. 1, p.195-208, 2012.

BERNARDINO, M. A.. D.; RODRIGUES, M. A.; BELLINI, L. M. Análise crítica das analogias do livro didático público de química do estado do Paraná. Ciência \& Educação, v. 19, n. I, p.135-150, 2013.

BRASIL, Ministério da Educação. Edital de convocação 01/2013-CGPLI - Edital de convocação para o processo de inscrição e avaliação de obras didáticas para o programa nacional do livro didático-PNLD 2015. Brasília: Ministério da Educação, Secretaria de Educação Básica, p.81, $2014 a$.

BRASIL, Ministério da Educação. Guia de livros didáticos: PNLD 2015 - Biologia: Ensino Médio. Brasília: Ministério da Educação, Secretaria de Educação Básica, p.79, 2014b.

BRASIL, Ministério da Educação. Parâmetros curriculares nacionais - PCN - Ciências. Brasília: 1997.

FRANCISCO, W.; FRANCISCO JUNIOR, W. E. A Bioquímica a partir de livros didáticos: um estudo dos livros de química aprovados pelo PNLEM 2007. In: XV Encontro Nacional de Ensino de Química (XV ENEQ). Anais... Brasília, 2007.

FRISON, M. D.; VIANNA, J.; CHAVES, J. M.; BERNARDI, F. N. Livro didático como instrumento de apoio para construção de propostas de ensino de ciências naturais. In: VII Encontro Nacional de Pesquisa em Educação em Ciências, Florianópolis. Anais... Santa Catarina: UFSC, 2009.

GOMES, H. J. P.; OLIVEIRA, O. B. Obstáculos epistemológicos no ensino de ciências: um estudo sobre suas influências nas concepções de átomo. Ciências \& Cognição, v.12, p.96109, 2007.

KATO, D. S; KAWASAKI, C.S. As concepções de contextualização do ensino em documentos curriculares oficiais e de professores de ciências. Ciência \& Educação, v. 17, n. 1, p. 35-50, 2011.

MIRANDA, F. A.; ARAÚJO, S. C. M. Identificação de obstáculos epistemológicos presentes em alguns livros didáticos de química do ensino médio. In: XVI Encontro Nacional de Ensino de Química (XVI ENEQ) e X Encontro de Educação Química da Bahia (X EDUQUI), Anais... Bahia: Salvador, 2012.

NELSON, D. L.; COX, M. Lehninguer - Principles of Biochemistry. $5^{a}$ Edição, Nova York: Freeman and Company, 2008.

NETO, J. M.; FRACALANZA, H. O Livro didático de ciências: problemas e soluções. Ciência \& Educação, v.9, n.2, p.147-157, 2003.

PIRES, A. S. Bioquímica no livro didático de ensino médio: um distanciamento da realidade do aluno? 2012, 41f. Trabalho de conclusão de curso (Licenciatura em Ciências Biológicas), Universidade Federal do Rio Grande do Sul, Porto Alegre.

ROSA, M. D'A. Seleção e uso do livro didático na visão de professores de ciências: um estudo na rede municipal de ensino de Florianópolis. 2013, 197f. Dissertação (Programa de 
Pós-graduação em Educação Científica e Tecnológica), Universidade Federal de Santa Catarina, Florianópolis.

SILVA, L. R. C.; DAMACENO, A. D.; MARTINS, M. C. R.; SOBRAL, K. M.; FARIAS, I. M. S. Pesquisa documental: alternativa investigativa na formação docente. In: IX Congresso Nacional de Educação (EDUCERE) E III Encontro Sul Brasileiro de Psicopedagogia, Anais... Paraná: Curitiba, 2009.

STADLER, J. P.; SOUSA JÚNIOR, F. S.; GEBARA, M. J. F.; HUSSEIN, F. R. G. S. Análise de obstáculos epistemológicos em livros didáticos de química do ensino médio do PNLD 2012. Holos, n.28, v.2, p.234-243, 2012.

TAUCEDA, K. C.; NUNES, V. M.; DEL PINO, J. C. O livro didático e as representações mentais de bioquímica e biofísica em alunos do ensino médio. Experiências em Ensino de Ciências, v.6, n.1, p.57-68, 2011.

ZAMORA, A. M. Obstáculos epistemológicos que afectan el proceso de construcción de conceptos del área de ciencias en niños de edad escolar. InterSedes: Revista de las Sedes Regionales, n. 3, v. 5, p.75-89, 2002. 